\title{
Le développement de dispositifs techniques de surveillance des espaces publics et la participation des habitants au maintien de l'ordre au Japon
}

Naoko Tokumitsu

\section{CpenEdition}

Journals

Édition électronique

URL : https://journals.openedition.org/cdg/7099

DOI : $10.4000 /$ cdg.7099

ISSN : 2107-7266

Éditeur

UMR 245 - CESSMA

Référence électronique

Naoko Tokumitsu, "Le développement de dispositifs techniques de surveillance des espaces publics et la participation des habitants au maintien de l'ordre au Japon », Carnets de géographes [En ligne], 15 | 2021, mis en ligne le 30 avril 2021, consulté le 28 mai 2021. URL : http://journals.openedition.org/ cdg/7099 ; DOI : https://doi.org/10.4000/cdg.7099

Ce document a été généré automatiquement le 28 mai 2021.

\section{(i) $९$

La revue Carnets de géographes est mise à disposition selon les termes de la Licence Creative Commons Attribution - Pas d'Utilisation Commerciale - Pas de Modification 4.0 International. 


\title{
Le développement de dispositifs techniques de surveillance des espaces publics et la participation des habitants au maintien de l'ordre au Japon
}

\author{
Naoko Tokumitsu
}

\section{Introduction}

1 Au Japon, dans les années 1970, l'Agence nationale de la police s'engage dans un changement de politique vis-à-vis de l'organisation du maintien de l'ordre dans les espaces urbains. Elle pointe un affaiblissement des liens de voisinage, qu'elle associe à l'urbanisation croissante du pays, à l'informatisation massive et à l'internationalisation de la société, qui auraient conduit à «la destruction des communautés locales qui avaient une force dissuasive " par rapport à la délinquance juvénile $»^{1}$. La police japonaise exprime ainsi la crainte que le système policier ne soit plus en phase avec les changements sociaux (Watanabe, 2005). Au milieu des années 1990, après avoir enregistré à plusieurs reprises les plus hauts taux d'infractions depuis la fin de la Seconde Guerre mondiale, la police japonaise se met à renforcer son volet préventif en étudiant les spécificités locales constatées des épisodes de délinquance. Dans le sillage des recherches anglophones (Clifford, 1976 ; Bayley, 1978) qui ont valorisé les actions des îlotiers à l'échelle locale, la police japonaise décide d'encourager cette forme d'intervention. Elle consolide alors progressivement son partenariat avec les collectivités locales pour intensifier la dimension locale de son activité de maintien de l'ordre $^{2}$. Cette nouvelle approche conduit la police à solliciter davantage la participation des habitants pour assurer le maintien de l'ordre et encourager un rapprochement sur le terrain entre les policiers et ces derniers, mais aussi à développer des outils techniques afin de faciliter la coopération entre forces de police et habitants ${ }^{3}$. 
Des aides sont fournies aux riverains afin qu'ils disposent de voitures à gyrophares pour leurs patrouilles, des lampadaires sont équipés de dispositifs de prévention afin de pouvoir informer rapidement la police en cas d'agression, des kōban (postes de police) civils sont mis en place, gérés non par des agents de police mais par des habitants et des commerçants locaux. Dans la lignée des modèles anglophones, la police japonaise développe ainsi une approche fondée sur la prévention situationnelle qui vise à «modifier de façon principalement défensive des situations que l'on juge génératrices de problèmes afin d'en atténuer la fréquence d'apparition ou la gravité (diminution des risques de commission d'une infraction et/ou augmentation des risques d'arrestation de l'auteur)» (Mary, $2001: 37$ ).

Dans cet article, nous nous intéresserons à la manière dont ces dispositifs ${ }^{4}$ urbanistiques et sécuritaires anglo-saxons ont été introduits et adaptés au contexte japonais. Il s'agira d'analyser comment ces éléments techniques et sécuritaires d'aménagement de l'espace ont été déployés, en nous intéressant plus particulièrement au renforcement des liens entre les autorités publiques et les organisations d'habitants. On cherchera à interroger les objectifs, les outils et les effets de la mobilisation des citoyens dans la co-production du maintien de l'ordre local. On montrera également comme cela participe à "délimiter les frontières de communautés imaginaires morales » (Fourchard, 2018).

Pour ce faire, nous détaillerons dans un premier temps l'évolution des doctrines de maintien de l'ordre produites par des instances gouvernementales, telles que l'Agence nationale de la police et les commissions interministérielles avant de nous intéresser aux dispositifs émergeant de ces doctrines. Nous mettrons d'abord en lumière les effets sur l'aménagement préventif des espaces publics. Puis, nous étudierons la manière dont ces réflexions ont renforcé la participation des habitants au maintien de l'ordre, en détaillant trois exemples: les patrouilles d'habitants, la cartographie participative et l'éducation morale des enfants.

\section{Encadré 1. L'enquête de terrain}

Afin de mener à bien cette étude, entreprise dans le cadre de notre travail de thèse (Tokumitsu, 2018), nous avons notamment analysé des sources administratives et législatives relatives aux politiques de prévention de la délinquance depuis les années 1970 : livres blancs publiés depuis 1973 par l'Agence nationale de la police, arrêtés, débats municipaux, rapports et dossiers ministériels, résumés d'activités de 352 groupes d'actions préventives, etc.

Nous avons aussi réalisé des enquêtes de terrain (observations directes et entretiens semi-directifs) menées entre 2016 et 2018 auprès de 52 personnes participant à ces actions (policiers, agents municipaux, riverains et élus locaux) à Tokyo, mais aussi à Sakai (Osaka) ainsi qu’à Kobe (Hyōgo).

Le choix de ces terrains de recherche est lié au constat de caractéristiques nettement différenciées selon les zones où sont déployées les actions. Les enquêtes ont été ainsi réalisées d'une part, dans des quartiers commerciaux marqués par une forte fréquentation de clients extérieurs, et d'autre part, dans des quartiers résidentiels où les commerces locaux sont notamment fréquentés par des habitants du quartier. Nous avons tout particulièrement réalisé des terrains au sein des quartiers se trouvant en banlieue ouest de Tokyo. Il s'agit de quartiers 
ayant pris la forme de villes dortoirs. Ce travail a été complété par des enquêtes de terrain au sein de quartiers de banlieue des villes de Sakai et Kobe. Ceux-ci, dont les caractéristiques diffèrent du cas tokyoïte, ont cependant été présentés comme des exemples d'actions préventives par l'Agence nationale de la Police. Dans ces deux cas, nous observons moins d'influence de la théorie de « vitre cassée ». Le cas de Sakai montre notamment une différence dans sa forme d'action essayant d'inclure les jeunes dits « voyous » au sein du groupe des habitants.

Ce travail de terrain a permis d'observer la manière dont les habitants réalisent leurs patrouilles en repérant les éléments ou individus considérés à risque, ainsi que les relations qu'ils entretiennent avec les agents policiers sur le terrain. Nous y avons pris part en tant que doctorante, extérieure à ces groupes préventifs. Dans ce présent article, nous abordons ainsi l'un des aspects étudiés, soit l'évolution des doctrines autour de la prévention situationnelle ainsi que la participation des habitants qui s'y associent.

\section{L'évolution des doctrines et politiques policières en matière de prévention de la délinquance depuis les années 1970}

Nous analyserons dans cette première partie l'élaboration d'une doctrine sécuritaire basée sur le remodelage de l'espace urbain et la mise en place d'une forme de «community policing» soulignant l'importance de la décentralisation des dispositifs de contrôle et la diversification des acteurs impliqués dans la coproduction de la sécurité qu'il s'agisse du secteur privé ou des habitants. Cette analyse des doctrines s'inscrit dans la continuité des acquis critiques de Joseph Gusfield qui montre, dans une approche constructiviste, que « reconnaître une situation comme pénible requiert un système de détermination et de catégorisation des événements " (2009: 3). Autrement dit, il s'agit d'interroger la manière dont, au Japon, la question de l'insécurité a été mise à l'agenda politique et s'est trouvée associée à la question du quartier et des liens entre habitants.

\section{La crainte d'un délitement du lien social et d'un affaiblissement du contrôle informel}

Dès les années 1970, avant de s'intéresser à la prévention situationnelle, l'Agence nationale de la police craignait le délitement des liens de voisinage, notamment à cause $\mathrm{du}$ déclin des communautés de quartier appelées chōnaikai ou jichikai ${ }^{5}$, un processus considéré comme allant de pair avec l'augmentation du nombre de grands immeubles et le changement de mode de vie urbain ${ }^{6}$. L'Agence nationale de la police associait en effet le développement de la délinquance juvénile à un contexte de transformation des communautés locales et notamment des structures familiales : cette transformation aurait "diminué les fonctions d'autogestion des problèmes, d'entraide ainsi que de dissuasion de la délinquance ${ }^{7}$. Notons qu'à cette période, entre la fin des années 1970 et le début des années 1980, la question des violences scolaires occupait une place importante dans les débats médiatiques ${ }^{8}$. Un peu plus tard, au milieu des années 1980 , 
l'Agence nationale de la police entendait faire des personnes âgées des acteurs bénévoles capables de revitaliser l'esprit de solidarité d'un quartier, affichant par là une visée de tissage du lien social ${ }^{9}$ (Tokumitsu, 2019). Autrement dit, à partir des années 1970, la police japonaise tâche progressivement d'élargir le champ de la police administrative dont les pouvoirs avaient été réduits au sortir de la guerre.

6 Le volet préventif de l'action policière s'est ensuite trouvé renforcé dans les années 1980 avec la réforme de la loi dite « füeihō» élargissant les objectifs policiers pour inclure des domaines tels que "l'éducation saine des adolescents" ainsi que des endroits tels que les centres de jeux. L'Agence nationale de la police avance ensuite, dans son livre blanc de 1994, qu'elle avait alors enregistré le plus haut taux d'infractions pénales, avec une augmentation générale des infractions impliquant des réseaux de délinquance interdépartementaux, ainsi qu'une augmentation des infractions commises par les arrivants étrangers. La même année, la réforme de la loi de la police (keisatsuhō kaisei) accorde des prérogatives préventives à la police, avec l'instauration d'un département de sécurité du quotidien (seikatsu anzenbu) au sein de l'Agence nationale de la police (Watanabe, 2005). L'objectif assigné à ce département consiste à assurer la sécurité et la tranquillité des habitants, notamment concernant des domaines tels que la vie quotidienne du quartier, la délinquance juvénile, les dégradations de l'environnement urbain.

\section{Les mises en application à l'échelle nationale de modèles importés}

7 C'est dans ce contexte que l'Agence nationale de la police japonaise commence à s'intéresser aux modèles de prévention situationnelle ${ }^{10}$ et que la politique de rapprochement entre police et population s'est trouvée particulièrement encouragée par la circulation de la notion de "community policing " ${ }^{11}$. L'Agence nationale a en effet étudié des politiques de réorganisation policière développées aux États-Unis ${ }^{12}$, au Canada $^{13}$, au Royaume-Uni ${ }^{14}$ et à Singapour ${ }^{15}$. Cependant, comme le soulignent Patrick Hassenteufel et Jacques de Maillard (2013: 380), de tels modèles n'ont pas exercé d'« effets mécaniques et directs sur les politiques nationales ». Ces modèles font l'objet de mises en application contrastées tout en s'inscrivant dans un même mouvement de diversification des acteurs du maintien de l'ordre également identifiable dans d'autres pays ${ }^{16}$. Dans le cas japonais, la notion de "community policing " a amené l'administration policière à promouvoir des postes de police fonctionnellement diversifiés, conçus non seulement comme des établissements administratifs mais également comme des lieux d'information et d'échange avec les habitants. En outre, l'Agence nationale de la police s'est intéressée aux modèles tels que le «Crime prevention through environmental design » aux États-Unis et en Angleterre (Shimizu, 2007), ce qui a également contribué à une forme de recomposition des espaces publics.

8 Cette évolution du rôle dévolu à la police dans la société est concomitante de la construction de l'insécurité en tant que problème public dans le discours des autorités. Deux évènements consécutifs font en effet de l'insécurité un thème central et entrainent la mise en place de plans successifs d'aménagement urbain inspirés de la prévention situationnelle. Le premier évènement est le tremblement de terre de Hanshin-Awaji du 17 janvier $1995^{17}$, année qui fut d'ailleurs appelée au Japon «la première année du bénévolat». Le second, survenu deux mois plus tard, le 20 mars 1995, est l'attentat au gaz sarin dans le métro de Tokyo ${ }^{18}$. 
9 Après l'attentat au gaz sarin, la police publie un sondage réalisé en décembre 1995 par le bureau du Premier ministre, selon lequel 63,3 \% des sondés ressentent de l'insécurité, $71,3 \%$ éprouvent une crainte à l'égard de problèmes qui ne relèvent pourtant ni du civil ni du pénal ; enfin, $88,7 \%$ des sondés estiment que la délinquance allait augmenter dans le futur ${ }^{19}$. L'influence des politiques développées dans les pays étrangers que nous avons évoquées plus haut s'observe tout d'abord dans un renforcement des contrôles de police, mis en œuvre suite à l'attentat au gaz sarin. La figure 1 montre une affiche placardée à Tokyo par la Fédération nationale des associations de prévention de la délinquance ${ }^{20}$ suite à cet événement porte ainsi l'inscription suivante : «Ce bagage vous appartient-il ? Ne soyez pas indifférents! Fabriquons ensemble une ville paisible» (Minna de tsukurō. Anshin no machi). En dessous de ces phrases et autour du personnage $\mathrm{du}$ chien $\mathrm{CP}-k_{u n}{ }^{21}$, nous pouvons observer les termes "crime prevention " indiqués en anglais. Bien que le message qui l'accompagne puisse varier en fonction de l'objectif des actions, ce personnage apparaît en effet non seulement sur des affiches dans les gares, mais il se trouve également utilisé dans des documents publiés par les associations de prévention de la délinquance, présentes dans 47 départements ainsi qu'au sein de commissariats au niveau local. Conçu en 1994 par le département de sécurité du quotidien (seikatsu anzenbu) au sein de l'Agence nationale de la police, ce personnage devient ainsi un symbole de changement doctrinaire au sein de la police japonaise, cherchant à impliquer la population dans les enjeux de prévention de la délinquance. Il semble fortement influencé par "McGruff the Crime Dog», créé en 1980 et promu par le Conseil de prévention du crime aux États-Unis.

Figure 1. Affiche représentant le personnage CP-kun

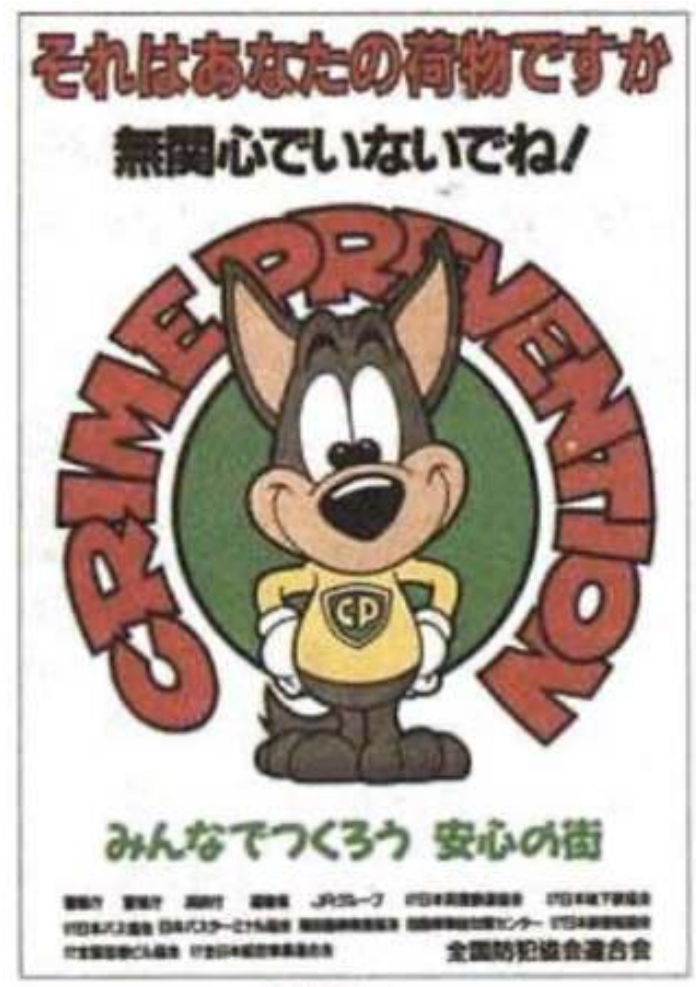

Source : site de la Fédération nationale des associations de prévention de la délinquance (zenkoku bōhan kyōkai rengōkai 全国防犯協会連合会), http://www.bohan.or.jp/ 
10 Il faut enfin évoquer un rapport publié par la police en janvier 1997, résumant les travaux d'un groupe de réflexion sur la sécurité quotidienne (seikatsu anzen kenkyūkai) de l'Agence nationale de la police ${ }^{22}$. Celui-ci insiste sur la nécessité d'impliquer la police dans des projets urbains, en faisant référence notamment à l'exemple du «Crime prevention through Environmental Design " (Shimizu, 2007). À partir de 2001, comme en témoigne un numéro spécial de la revue "Nikkan keisatsu», destinée aux agents policiers, deux piliers de ces mesures préventives sont soulignés: d'une part, une approche prônant le renforcement de la présence humaine dans la ville et la promotion des entreprises privées de sécurité, et d'autre part une approche plus urbanistique visant à aménager les espaces urbains eux-mêmes (Ibid.).

11 Avec la concrétisation de ces mesures, la police et le gouvernement placent les enjeux sécuritaires au centre de leur action. Les actions préventives sont dès lors présentées dans les commissions interministérielles comme un enjeu national impliquant tous les ministres. Ces commissions de lutte contre la délinquance se réunissent en 2003, à l'initiative de l'office du Cabinet, autour du mot d'ordre suivant: "Pour ranimer le Japon, "pays le plus sûr au monde" ". Sollicitant la participation de chaque ministère, ces échanges associent notamment le constat d'une aggravation de la délinquance avec l'arrivée de "visiteurs étrangers $»^{23}$ (rainichi gaikokujin) dont le taux d'infraction, d'après la police japonaise, était supérieur à celui des personnes catégorisées comme japonaises ${ }^{24}$. Parallèlement à cela, l'Agence nationale de la police lance une réforme du système budgétaire basée sur la méthode en quatre étapes dite «Plan, Do, Check, Act » (PDCA), axe important de la nouvelle gestion publique (NPM) introduite par le gouvernement. Selon cette approche, le budget alloué à la police est conditionné à l'obtention de résultats chiffrés, c'est-à-dire à une réduction du taux de criminalité constatée.

12 Cette nouvelle politique insiste sur l'importance de la coopération entre police et habitants, afin que ces derniers n'ignorent pas le fait que des infractions sont commises au sein de leur quartier, même s'il s'agit de faits mineurs. Ces mesures politiques se trouvent ensuite justifiées par la théorie de la «vitre cassée » ${ }^{25}$ selon laquelle le moindre acte déviant peut dégrader l'ordre public tant sur le plan de l'aménagement du quartier que pour les comportements des individus.

13 Ces éléments montrent que la police japonaise élargit son volet préventif, en croisant les enjeux sécuritaires avec les enjeux urbains. Cette orientation se trouve également justifiée par la menace ressentie à l'égard de la sécurité du pays, ce qui entraine notamment une évolution des doctrines policières aussi bien au niveau national que local, contribuant à inclure la population dans la gestion de la sécurité comme de la tranquillité publique.

\section{Élargir le contrôle du quotidien par l'aménagement de l'espace}

Cette partie interroge la manière dont ces politiques de prévention se sont traduites dans des projets d'aménagements des espaces publics ainsi que les modalités d'implication des acteurs de terrain, tout en prêtant attention aux différences qui apparaissent selon les quartiers. Cette évolution doctrinaire, qui a pour objectif affiché de créer une «ville sûre et paisible » et de renforcer la "force du quartier » (voir ci- 
dessous), se traduit par des dispositifs d'occupation et d'aménagement de l'espace public, tels que la mise en place de « super lampadaires » qui contribuent à chasser les personnes jugées déviantes ou indésirables.

\section{Des dispositifs techniques pour soutenir la fabrique de la ville « sûre et paisible »}

Entre 1997 et 1999, des réunions consacrées à la prévention situationnelle sont organisées par le ministère de la Construction ${ }^{26}$ (kensetsushō) et l'Agence nationale de la police. Celles-ci réunissent des spécialistes impliqués dans la conception de projets urbains : urbanistes, professeurs d'université spécialisés dans la gestion des désastres naturels, professeurs de criminologie et de droit, ou encore cadres policiers ainsi que responsables administratifs en matière de parc immobilier public. Les réflexions sont conduites à partir d'observation des actions menées dans quatre villes différentes, choisies comme "villes modèles $"^{27}$ : une ancienne zone industrielle en plein renouvellement urbain de la ville de Kawasaki, la ville de Kobe qui a subi les grands tremblements de terre de 1995, Neyagawa qui connait une forte concentration de maisons en bois, et enfin un quartier résidentiel ainsi qu'un grand terrain vague abandonné par une entreprise dans la ville de Himeji ${ }^{28}$. Les actions menées dans ces quatre villes s'inscrivent dans les politiques nationales sous le slogan de «fabrique de la ville (machi-zukuri) sûre et paisible », et impliquent l'amélioration de l'environnement en vue de prévenir la délinquance mais aussi les catastrophes, les accidents de la route ou encore de mieux organiser l'action sociale. Des questions furent notamment soulevées concernant les enjeux de la prévention de la délinquance et ceux de la prévention des catastrophes naturelles qui peuvent être contradictoires: si, par exemple, la première s'accompagne d'une forme de fermeture des bâtiments visant à assurer la sécurité de l'intérieur, la second exige au contraire de faciliter la sortie en cas de catastrophe. De même, si la prévention des catastrophes peut justifier la plantation d'arbres spécifiques pour empêcher la propagation d'un feu, la réflexion sur la sécurité s'y oppose dans la mesure où cela pourrait créer un espace sombre, menaçant pour les passants, créant une potentielle zone d'insécurité. Ces débats ont été vifs notamment dans le cas des villes de Kobe et de Neyagawa, où les habitants se sont davantage inquiétés des risques de catastrophe après avoir subi le tremblement de terre de Hanshin-Awaji. Des débats publics cherchent alors à déterminer comment l'aménagement de l'espace peut concilier ces deux enjeux. Les experts participant à ces réunions essaient de trouver des solutions en étudiant à la fois les conditions matérielles (serrures, hauteur des murets en parpaings, aménagements des espaces publics, etc.) et sociales, en prenant en compte le rôle des communautés locales. Ces dernières sont en effet considérées - par les riverains et les autorités publiques comme les premiers acteurs concernés en cas de catastrophes, ce qui conduit à insister sur leur rôle en matière de sécurité locale. Ces réunions aboutissent en 2001 à la publication d'un document intitulé «Livret de la fabrique de la ville sûre et paisible (anzen anshin machi-zukuri)». C'est à partir de cette période que l'expression de «ville sûre et paisible " a commencé à être largement employée au Japon (Amemiya et al., 2006). Cette expression articule des enjeux à la fois sécuritaires, urbanistiques et de prévention des catastrophes, mais en appelle aussi à des relations sociales pacifiées. 
16 L'Agence nationale de la police japonaise souligne, à travers les expériences menées dans ces 4 villes, la nécessité de s'adapter aux spécificités locales tout en s'intéressant à des expériences étrangères de déploiement de nouveaux dispositifs préventifs, notamment au projet "Secured by Design", ou encore à un service du SCAS (Serious Crime Analysis Section de la National Crime Agency) qui établit des bases de données recensant les lieux jugés dangereux dans les villes britanniques ${ }^{29}$.

Le déploiement de la vidéosurveillance est également encouragé dans ce cadre. Les questions des droits de l'homme ou de la violation de la vie privée sont ainsi présentées comme secondaires par rapport aux avantages de tels dispositifs et les arguments liés au coût d'une telle technologie apparaissent avoir été rapidement écartés :

On dit que la vidéosurveillance ne se développe pas à cause de son coût, mais je pense qu'il faut placer des caméras aux endroits adéquats pour la prévention. Sur la question de la vie privée, je n'ai jamais entendu parler de cas où les données vidéo ont été utilisées de manière inappropriée et où les droits de l'homme ont été violés. Je crains qu'on utilise trop facilement des termes comme vie privée ou droits de l'homme. Si cela peut servir à la prévention de la délinquance, il est nécessaire de pouvoir vérifier les données dans le cas où il y aurait vraiment une violation grave. (Propos tenus par un professeur de droit lors d'une réunion sur la prévention situationnelle en $1998^{30}$ ).

S'ensuivent des réflexions portant sur la possibilité technique d'activer les caméras uniquement lorsqu'il y a un risque. Ainsi, si la question du respect de la vie privée n'est pas absolument absente des débats, la priorité est de mieux protéger les individus des infractions, mais aussi d'éviter qu'ils n'en soient victimes.

La focalisation des débats sur la prévention situationnelle s'observe également dans les plans d'aménagement. Outre la question de la vidéosurveillance, ces réunions se sont penchées sur quatre principaux axes ${ }^{31}$. Le premier correspond à une stratégie de renforcement de la protection, visant à faire disparaître - ou à renforcer - les objets susceptibles d'encourager la délinquance : il s'agit par exemple de remplacer les murs de béton par des haies afin d'éviter les graffitis. Le deuxième axe consiste à éloigner les malfaiteurs, par exemple en éliminant les points d'appui afin de les empêcher d'entrer dans les logements. Le troisième axe vise à améliorer la visibilité dans les espaces publics. Ainsi, la construction de murs en parpaings, fréquente en zone résidentielle, fut remise en question au nom de la sécurité, à la différence des grilles ou des haies permettant tout à la fois de dégager la vue et de compliquer les déplacements furtifs. Tous ces points qui concernent l'aménagement urbain amènent enfin la police à insister sur l'importance des échanges entre habitants. Ces réunions ont ainsi conduit à souligner les risques que peut causer un repli individuel : l'isolement des individus, la perte d'échanges entre habitants, ou encore la baisse de la «force du quartier» (chiiki no chikara), expression qui désigne l'autonomie et la participation des membres des communautés locales en vue de se protéger par eux-mêmes contre les risques sur un plan sécuritaire ou socio-sanitaire. Les participants aux réunions recherchent ainsi de nouvelles modalités d'action pour composer avec une société privilégiant l'individu, le repli sur soi, ainsi qu'un certain anonymat. Les conclusions de ces réunions sur la prévention situationnelle insistent finalement sur l'importance de faire participer activement les habitants à la vie locale par divers moyens : entretien des haies afin de montrer une présence, organisation d'événements locaux, et participation à la gestion des parcs, afin de renvoyer l'image d'un quartier auquel les habitants se montrent attentifs. 


\section{Le cas des super-lampadaires : développer les échanges entre certains habitants et en exclure d'autres}

20 L'importance accordée par les politiques sécuritaire et urbaine à la communication entre habitants, collectivités locales et police, peut s'observer à travers l'installation d'équipements au sein des espaces urbains et péri-urbains. Analysons à cet égard l'exemple des «super-lampadaires" (supā bōhantō) : en 2001, 190 lampadaires ont été installés par l'Agence nationale de la police dans 10 quartiers $^{32}$. Ceux-ci assurent non seulement une fonction d'éclairage, mais également d'alarme d'urgence, de vidéosurveillance, d'interphone permettant de communiquer avec la police et de lui transmettre les images des environs (voir figure 2). Ceci diffère du système de vidéosurveillance installé dans les zones commerçantes telles que le centre-ville de Shibuya ou de Shinjuku, dont les images sont destinées à des agents chargés de ce service au sein du commissariat du secteur correspondant ainsi qu'à l'Agence nationale de la police, et qui se tiennent à disposition 24 heures sur 24 afin de réagir au plus vite aux éventuels faits jugés dangereux (Yamamoto, 2005).

Figure 2. Schéma d'un « super-lampadaire »

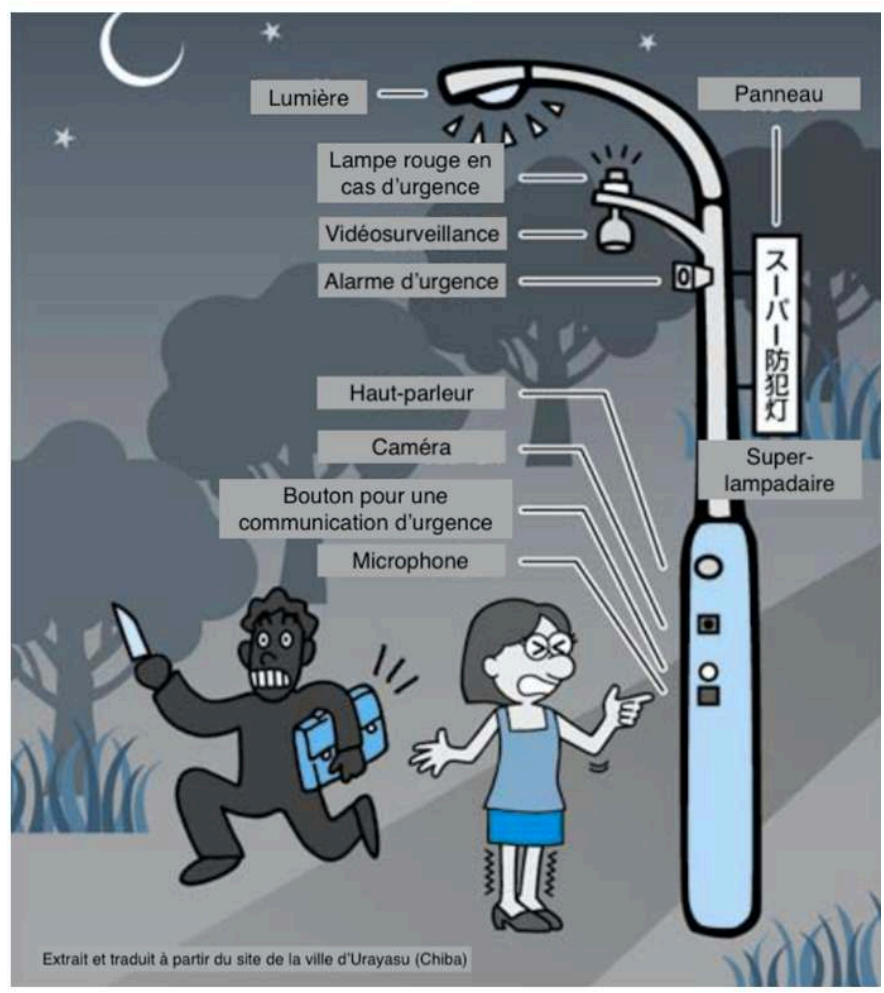

Source : site de la ville d'Urayasu (Chiba), schéma traduit par l'auteure

Les technologies telles que les super-lampadaires sont conçues comme des moyens pouvant d'une part remplacer la présence des postes de police et d'autre part, renforcer l'attachement au quartier des habitants en favorisant les échanges, et en développant le sentiment de sécurité par l'éclairage public ${ }^{33}$. En outre, ces superlampadaires sont présentés comme adaptés aux besoins d'une société vieillissante ayant un faible taux de natalité. Ils s'inscrivent dans la perspective du projet 
économique de "ville compacte $»^{34}$ dont l'objectif est là aussi d'esquisser un plan de fabrique de la ville "sûre et paisible » : en concentrant la population en un périmètre restreint réunissant toutes les commodités essentielles de la communauté (commerces, hôpitaux, écoles, crèche, centres de loisirs et lieux de travail), il s'agit de promouvoir davantage l'économie locale et de mieux faire face aux risques de désastres naturels ou d'infractions en resserrant le lien avec la police. Résultant d'un partenariat entre le ministère de la construction et l'Agence nationale de la police, ce projet est notamment lié à la crainte de la décroissance démographique au sein des banlieues ainsi qu'à l'idée que le maintien de l'ordre contribue à l'animation et à la prospérité du quartier. Précisons enfin qu'il s'agit là d'un dispositif expérimental mis en œuvre de façon locale et temporaire, et qui a fait l'objet de critiques concernant son coût.

Cette première expérimentation a toutefois suscité l'intérêt d'autres municipalités. Ainsi, plusieurs villes ont cherché à améliorer l'éclairage public pour prévenir la délinquance. Dans un quartier d'okinawa, le projet «stratégie light up » (raito appu sakusen), lancé en 2001, reposait sur une enquête policière qui aurait montré que plus de $60 \%$ des actes délinquants ou criminels surviennent dans des endroits mal éclairés (Yamamoto, 2005). Chaque habitant du quartier a ainsi cotisé la somme symbolique de 110 yens (environ $1 €$ ) afin d'améliorer l'éclairage public. Par ailleurs, des enquêtes interministérielles ont été organisées par le Cabinet du Premier Ministre afin d'examiner l'aménagement des rues que les enfants empruntent pour aller à l'école. Ces enquêtes soulignent aussi l'importance de proposer des lieux où les personnes handicapées ou âgées puissent se reposer, afin de garantir la sécurité des enfants, ces derniers étant alors entourés de nombreux habitants qui les regardent et veillent sur eux $^{35}$ (Ibid.). L'espace visible est ainsi élargi dans un objectif sécuritaire, par un renforcement des réseaux de surveillance de jour comme de nuit, souvent en invoquant l'aspect esthétique de la ville ou le renforcement des liens de solidarité à l'échelle locale.

23 Toutes ces mesures mettent ainsi en avant l'importance de produire des lieux « sûrs et paisibles » et non pas seulement de surveiller des personnes considérées « à risque ». La politique de prévention de la délinquance a ainsi évolué en développant des dispositifs techniques de surveillance et en les associant à d'autres enjeux, notamment ceux de prévention des dégâts causés par les catastrophes ainsi que d'animation de la vie locale par les échanges entre voisins. Cependant, nous avons observé que ces aménagements d'espaces urbains se sont également accompagnés de formes d'exclusions, qui diffèrent toutefois selon les caractéristiques géographiques des espaces considérés. Nous pouvons dès lors interroger la manière dont les actions préventives se sont développées dans les zones résidentielles, en analysant les différents outils permettant de renforcer la participation des habitants dans le cadre du développement d'une forme de community policing.

\section{La participation des habitants au « community policing »}

Comme nous l'avons montré plus haut, les orientations politiques de la prévention de la délinquance ont été consolidées dans les années 1990 avec l'importance accordée aux échanges entre voisins. Les années 2000 se caractérisent par la préoccupation à l'égard des criminels étrangers et la mise en œuvre d'une politique du chiffre soulignant 
l'importance de la diminution du taux d'infractions, mais aussi par une forte augmentation de la participation des habitants aux actions préventives. D'après les statistiques établies par l'Agence nationale de la police japonaise, environ 180000 personnes participaient à ces actions en 2003, tandis que 5 ans après, en 2008, elles étaient environ $2500000^{36}$. William Skogan (2006) a retenu, concernant le community policing, trois principales caractéristiques : 1) La décentralisation, 2) L'implication des communautés, 3) La résolution des problèmes. Nous montrerons dans cette partie les adaptations de ce modèle anglophone au contexte japonais, en étudiant différents outils mobilisés pour renforcer la participation des habitants dans la coproduction de la sécurité: les patrouilles d'habitants et leurs voitures à gyrophare, ainsi que la cartographie participative. Nous nous intéresserons ensuite au volet pédagogique et moral de cette approche.

\section{Les patrouilles d'habitants et le cas des voitures à gyrophare} groupe se donnent rendez-vous puis déambulent au sein d'un périmètre prédéfini, correspondant souvent à l'entité administrative où se trouve leur résidence. Pendant les patrouilles, certains discutent entre eux, apprécient la verdure, saluent des personnes qu'ils croisent au détour d'une rue, ou encore ramassent des déchets laissés au sol. Les groupes organisant des patrouilles d'habitants sont en général formés à l'initiative de résidents qui ont notamment été victimes de cambriolage ou d'une diminution du chiffre d'affaire de leurs commerces et qui donc partagent les craintes que nous retrouvons dans les propos des policiers et dans les médias. Beaucoup d'entre eux entretenaient déjà un rapport étroit avec des agents de police locaux, soit à travers les réseaux des chōnaikai évoqués plus haut, soit via des activités commerciales de quartier. Les agents de police ne participent pas d'ordinaire aux patrouilles d'habitants ni à leurs réunions régulières.

Ces mobilisations peuvent être considérées comme "semi-autonomes", selon la distinction établie par Les Johnston (1996) entre mobilisations "autonomes" (entreprises sans l'accord de l'État) et mobilisations «encadrées " par l'État. Car ce dernier, dans le cas japonais, encourage et continue à soutenir a posteriori ces mobilisations. Pour soutenir ces dispositifs, l'Agence nationale de la police a par exemple conçu un «plan de renouvellement pour une société locale forte contre la délinquance ${ }^{{ }^{37}}$ et des directives gouvernementales ont encouragé la police et les collectivités locales à concrétiser leur soutien en fournissant un équipement (lampes, brassard, gilet, papeterie, etc.), un lieu de rassemblement (un local, par exemple un centre municipal) et éventuellement des gyrophares destinés aux patrouilles. La police considère que les actions bénévoles des habitants contribuent pleinement au maintien de l'ordre local ce qui transparaît par exemple dans un journal tenu par un policier, évoquant un travail mené au début des années 2000 :

«Quand on m'a nommé chef du service de la sécurité du quotidien en 2003, la politique de la prévention de la délinquance venait de commencer. À l'époque, il fallait réduire en trois ans le taux de délinquance au niveau de celui atteint dix ans plus tôt. Je pensais que ce n'était pas réalisable par la police seule mais, à ce moment-là, est apparu le groupe A (groupe d'habitant anonymisé). À l'époque, j'ai eu l'impression que vous [le groupe d'habitant] étiez arrivé comme un sauveur.» (Propos écrits par un ancien policier dans un journal d'activités du groupe d'habitants concerné, juillet 2008 ${ }^{38}$ ) 
démarche se distingue du neighborhood watch né aux États-Unis à la fin des années 1960, dont les activités sont animées par des coordinateurs formés par les policiers (Jobard et Maillard, 2015) et sont soutenues, depuis 1972, par l'association nationale des shérifs (National Sheriffs' Association). Au contraire, dans la majorité des cas, au Japon, il n'existe pas de processus de délibérations entre acteurs publics et riverains. Cela se distingue ainsi de ce qu'avait remarqué Archon Fung (2000) à propos des organisations communautaires à Chicago, où les problèmes de sécurité publique sont identifiés de manière collective, de façon à définir les priorités les plus urgentes pour le quartier.

L'utilisation des voitures avec gyrophares par les collectifs d'habitants constitue un autre exemple intéressant afin d'illustrer la manière dont les actions des habitants ont été encouragées par les autorités publiques. Jusqu'en 2004, la loi relative à la circulation et aux transports n'autorisait l'usage d'un gyrophare qu'aux véhicules d'urgence officiels. Mais les autorités publiques ont souhaité modifier ces lois, pour répondre aux demandes des habitants et des collectivités voulant couvrir une plus large zone de patrouille. Les discours des acteurs de terrain mettent d'ailleurs en avant l'utilité des voitures de patrouilles d'habitants équipées d'un gyrophare, qui permettent de poursuivre un conducteur prenant la fuite :

La ville de Machida est comme un appendice. Ici, il y a le département de Kanagawa dont la ville de Kawasaki est voisine. Et quand il y a une frontière, c'est difficile de contrôler. Il est facile de commettre une infraction. Puis, au sud de Machida, il y a une autoroute et deux grandes lignes ferroviaires. Donc il est très facile de prendre la fuite.

(Entretien avec le vice-président d'une communauté de quartier, septuagénaire, le 22 février 2016)

L'élargissement du champ des autorisations sous certaines conditions ${ }^{39}$ a ainsi répondu aux attentes des habitants voulant contribuer au maintien de l'ordre. Ces derniers réalisaient déjà des patrouilles en voiture en indiquant sur une affichette le nom de leur communauté de quartier (débats parlementaires de 2004 ${ }^{40}$ ). La figure 3 présente un véhicule non policier muni d'un gyrophare. 
Figure 3. Voiture à gyrophare (ao-pato) gérée par des habitants à Tokyo

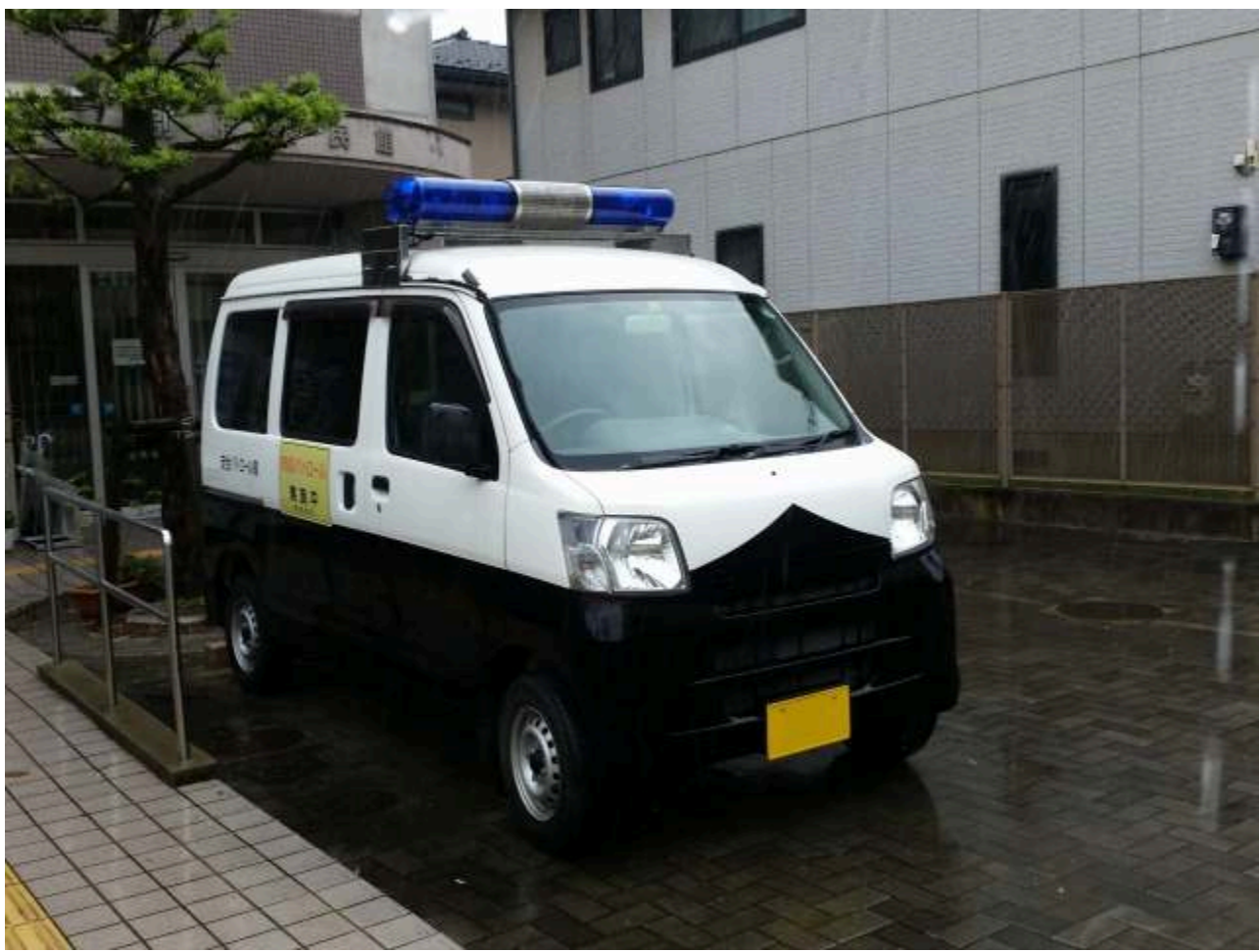

Source : Photographie libre de droit (photo AC)

Les statistiques publiées par l'Agence nationale de la police montrent que si, en 2004, on dénombrait 120 voitures utilisées par 102 groupes, ce chiffre a considérablement augmenté en 10 ans. Ainsi, en 2014, 43976 voitures autorisées étaient utilisées par 9470 groupes, ainsi que l'on peut le voir sur la figure 4 .

Figure 4. Augmentation du nombre de voitures à gyrophare (en orange) et du nombre de groupes qui y ont recours (en bleu)

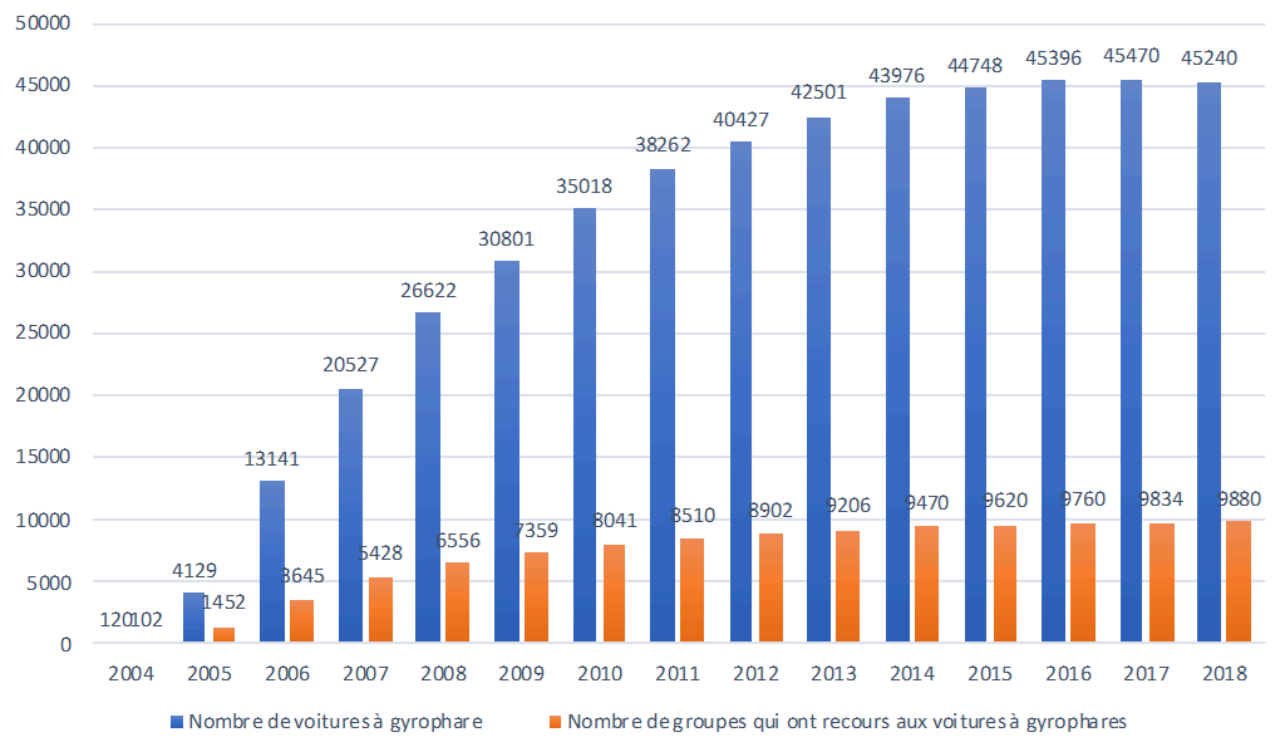

Source : Statistiques publiées par l'Agence nationale de la police sur les habitants participant activement aux groupes préventifs, mars 2018 
31 Les habitants engagés dans la mise en œuvre de ces patrouilles étaient majoritairement, en décembre 2019, des personnes âgées, 70 \% d'entre eux ayant plus de 60 ans. Il s'agit donc bien d'une classe d'âge désignée par l'Agence nationale de la police comme de potentiels acteurs bénévoles contribuant à la revitalisation de la solidarité locale, suivant la doctrine établie dans les années 1990. Le contenu des activités est fixé en fonction des groupes mais la majorité d'entre eux réalisent des patrouilles et veillent sur les enfants, sur le chemin de l'école. Cet élargissement du périmètre des patrouilles qui portent une attention particulière à l'égard des enfants, est facilité par cette voiture à gyrophare bleue qui, comme en témoignent de nombreux participants interrogés, s'avère très populaire auprès des plus jeunes: ce véhicule permettrait ainsi une identification plus claire ainsi qu'un rapprochement plus facile avec les enfants.

32 L'attention que les habitants portent à des endroits peu visibles et dégradés qui seraient susceptibles d'alimenter le sentiment d'insécurité peut s'accompagner d'un repérage des individus jugés suspects, voire d'une stigmatisation de ces derniers. Autrement dit, cela a aussi pour conséquence l'exclusion de ceux considérés comme déviants, des personnes jugées "errantes", comme les bandes de jeunes dans les espaces publics (Sibley, 1995). Cette dynamique d'exclusion s'est par exemple manifestée à partir de 1999 à l'encontre des personnes sans domicile fixe ; elle aurait alors eu «des effets sur la sécurité et la tranquillité du quartier, comme sur le sentiment d'insécurité des habitants du quartier ${ }^{41}$. Dans ce cadre, les policiers ont été incités par leur hiérarchie, d'une part, à renforcer les patrouilles afin de mettre fin à des agissements considérés comme propres à susciter le sentiment d'insécurité et, d'autre part, à prendre en charge si nécessaire les personnes concernées en les orientant vers des institutions d'action sociale afin d'encourager leur insertion professionnelle, en partenariat avec les collectivités locales. Il en va de même pour les jeunes vus comme "déviants». À titre d'exemple, des groupes de commerçants en contexte urbain, comme à Shibuya (Tokyo), craignant un impact négatif sur l'image de leur quartier, se sont mis à patrouiller en repérant les endroits qui leur semblent dégradés ou sales. Ces patrouilles ont ainsi abouti, au nom de la prospérité économique, à légitimer des actions visant à écarter des groupes de jeunes qui se retrouvaient au sein du quartier.

33 Ces formes d'exclusion peuvent varier en fonction des traits caractéristiques des quartiers et des priorités locales. On peut à ce titre s'appuyer sur la distinction opérée par Franck Furstenberg (1971) entre la peur personnelle d'être victime d'un crime et la peur générale que des crimes puissent être commis : nos entretiens semi-directifs avec le chef et le sous-chef d'un groupe préventif ainsi que l'analyse des débats municipaux montrent en effet que, si à Shibuya les actions sont menées au nom de crimes pouvant engendrer une peur généralisée au sein du quartier, notamment autour de la question des arrivants étrangers, dans les zones résidentielles en banlieue de Tokyo, les actions apparaissent davantage motivées par la peur d'être victime ou par la crainte que les enfants du quartier ne soient victimes de la criminalité. Ainsi, les patrouilles sont d'abord réalisées à l'initiative d'habitants se sentant en insécurité, avec le soutien d'agents de police, qui s'appuient à leur tour sur ces actions pour déployer des réseaux de surveillance favorisant la collecte d'informations. Ceci s'accompagne d'un soutien matériel des autorités publiques, qui fournissent par exemple brassards, vêtements, ou voitures, légitimant la présence des patrouilles au sein des espaces publics. Cela conduit 
à interroger les relations entre ces participants et les autorités publiques, notamment en matière d'informations relatives aux délits constatés. Ceci permet également d'observer de quelle façon les habitants mobilisent les outils dont ils disposent et comment leur attention se porte sur certaines catégories de population.

\section{Cartographie participative et repérage des individus « à risque »}

le cadre de discussions informelles ou lors de patrouilles, les policiers fournissent des indications à certains habitants (notamment aux responsables des communautés de quartier ou des établissements scolaires) afin qu'ils repèrent les lieux dangereux et renforcent les patrouilles au sein de leur quartier. À ceci s'ajoute une liste de diffusion pour transmettre des informations par courriel aux habitants qui y sont abonnés. Ces outils leur permettent d'accéder à des informations sur les formes de délinquance constatées, sur les activités préventives et sur les enquêtes policières en cours, ainsi que sur des techniques de prévention contre la délinquance (notamment contre l'escroquerie par téléphone, les agressions sexuelles, les enlèvements d'enfants). Les messages peuvent être envoyés dès qu'une affaire survient dans le secteur choisi par les abonnés et sectorisés en fonction du périmètre de chaque commissariat. Selon notre analyse des courriels envoyés aux habitants par le Département de police métropolitaine de Tokyo entre le 23 juillet et le 29 décembre 2016, les affaires signalées concernent surtout les enfants, les femmes et les personnes âgées. Ainsi, parmi les 212 signalements relayés par la police, 109 concernent des agressions de rue sur des enfants (coups, agressions verbales ou attouchements sexuels), 59 des comportements obscènes dans l'espace public, 23 des agressions de rue ou des cas de rôdeurs («stalkers »), 8 des vols à l'arraché, 4 des arnaques visant des personnes âgées, et 3 la recherche d'enfants ou de personnes âgées disparus. La physionomie de personnes considérées comme «suspectes" est détaillée à travers la description de caractéristiques physiques significatives (les cheveux, des vêtements, l'âge, etc.). En voici un exemple :

Le 1er septembre, vers 18h30, dans la rue XXX (adresse approximative du lieu), un homme a adressé la parole à une élève qui passait : « Ça te dirait de faire l'amour?» Il aurait environ 20 ans, porterait un T-shirt blanc et un pantalon noir et roulerait avec un vélo de ville de couleur argentée.

Message envoyé par la police de Tokyo, le 1er septembre 2016 à $21 \mathrm{~h} 35$

36

est survenu un délit, comme le montre la figure 5. 
Figure 5. Carte accompagnant un message de la police, indiquant la zone où est survenu un délit

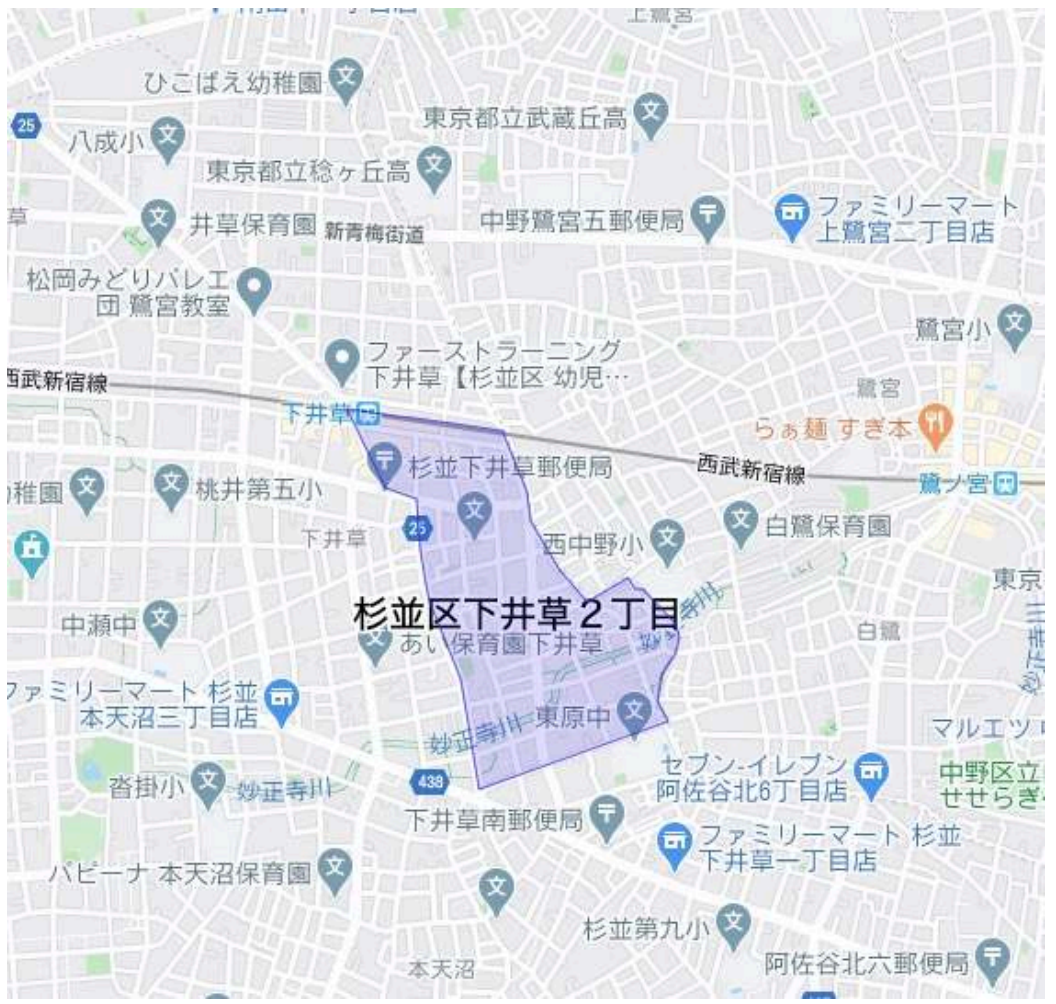

Source : Courriel envoyé par la police de Tokyo, février 2018

37 Comme l'a remarqué Melina Germes, la cartographie policière ne constitue pas un miroir de la réalité mais permet le plus souvent de «mobiliser l'image pour confirmer une représentation existante, mise visuellement en évidence » (2014, p. 21), et ce en vue de trois principaux enjeux territoriaux: «la construction de l'identité de l'institution dans l'espace; la construction de l'adversité dans l'espace; l'enjeu de la maîtrise de l'espace » (ibid.). Concernant notre cas d'étude, la mise à disposition de ces cartes a pour effet de renforcer le sentiment de légitimité des habitants, notamment des chefs de groupes préventifs, et cela les incite à accentuer les patrouilles dans les zones présentées comme dangereuses. L'attention tend ainsi à être dirigée vers des individus visiblement peu connus ou peu intégrés au sein du quartier, tels que les jeunes célibataires ne faisant souvent pas partie des organisations de voisinage et avec lesquels les acteurs de groupes préventifs n'ont pas l'occasion d'échanger au quotidien. Des différences de comportement par rapport à d'autres personnes reconnues comme membres de la communauté permettent de mobiliser une forme d'intuition - ou de préjugés. Un de nos enquêtés, policier retraité et chef d'un groupe préventif, s'en explique ainsi :

«On parlait à l'époque d'organisations criminelles venant de Chine et de leurs cambriolages... En réalité, on ne peut pas savoir s'il s'agit d'un Chinois ou d'un Japonais à première vue. Mais au fur et à mesure des expériences, nous avons pu juger en fonction de leurs comportements... C'est l'accumulation d'expériences qui a permis cette intuition. »

(Entretien avec le chef d'un groupe préventif à Tokyo, le 31 juillet 2017).

En complément de ses rôles administratif, d'analyse et opérationnel (Germes, 2014), la cartographie policière vise à renforcer le lien entre police et habitants et à amener ces derniers à s'auto-surveiller et à prêter attention à l'apparence de chaque individu. Les 
habitants évoquent également la portée motivationnelle de ce partage d'informations, qui était importante notamment lors du démarrage des actions : ils estiment que les chiffres de diminution ou d'augmentation d'infractions au sein du quartier, transmis par les agents de police locaux, leur permettaient de mesurer la portée de leurs activités quotidiennes notamment dans la période où ils mettaient en place les actions; ils s'appuyaient donc sur ces données pour échanger avec les habitants d'autres quartiers à propos des difficultés rencontrées ou des moyens d'empêcher les infractions.

Enfin, cet élargissement des réseaux de voisinage s'accompagne, notamment depuis février 2006, d'une réflexion sur le système de prévention déployé au sein de la ville, réflexion jugée nécessaire pour la prévention des catastrophes. Au nom des « réseaux d'information ubiquitaire ${ }^{43}$, ce dispositif vise à suivre les déplacements des enfants, par exemple à travers la fonction GPS du téléphone portable, et encourage à alerter si nécessaire les voisins pour leur demander secours. L'idée est ainsi de créer des réseaux de protection en vue de la prévention de la délinquance et des catastrophes.

\section{L'éducation morale des enfants : les effets escomptés de la sociabilisation et des programmes éducatifs}

Le développement de la cartographie contribue à la production d'une conscience collective des enjeux sécuritaires mais également à la légitimation des actions préventives associées à ces dispositifs et encouragées par les autorités publiques. Lorsque ces actions ont obtenu une reconnaissance au sein du quartier, les outils numériques constituent aux yeux des habitants interrogés des moyens relativement complémentaires de leur action visant à augmenter les échanges entre voisins et renforcer le contrôle social informel à l'échelle de la communauté. Ces habitants confirment la nécessité d'une protection des enfants du quartier par une présence humaine. Ils soulignent ainsi l'importance de saluer les passants à haute voix et de leur propre initiative, afin d'éloigner les personnes qui refuseraient de le faire pour ne pas être repérées. Cette prévention par la sociabilité est considérée, notamment par des membres des groupes préventifs en quête d'appartenance au sein du voisinage, comme une technique plus efficace que les équipements de vidéosurveillance ou les alarmes, ou encore que la seule présence des policiers. Cette tendance s'observe plus fortement au sein des quartiers dont la préoccupation principale ne porte plus sur les cambriolages mais sur le déclin de la sociabilité en raison d'une population vieillissante. Craignant que cela n'entraîne des effets néfastes non seulement pour leur sécurité mais également en matière d'éducation, ces habitants enseignent aux enfants l'importance de saluer, en entendant par-là que les jeunes qui ne le font pas peuvent être assimilés à des figures de « voyous » ou à des personnes mal intentionnées.

«[...] J'apprends trois choses aux enfants: le sourire, le sens du défi et le remerciement. Il faut dire bonjour à n'importe quelle personne qu'ils croisent. Je ne dis pas qu'il ne faut pas saluer un inconnu. Ce qui est important, c'est de ne pas se laisser perdre par soi-même. Mener cette action, c'est la bataille de chacun. »

(Entretien avec le président du chōnaikai à Kobe, le 5 mars 2016)

41 Enfin, l'accent mis sur les échanges entre voisins s'observe dans un dispositif d'éducation dite participative des enfants, à travers la création d'un "plan de sécurité » (anzen mappu), fortement encouragé dans les écoles primaires par le ministère de l'Éducation, de la Culture, des Sports, des Sciences et de la Technologie. Il 
s'agit de faire connaître aux enfants les lieux jugés dangereux à éviter mais également de leur faire rencontrer des personnes âgées du quartier. Les enfants en bas âge, notamment ceux de l'école primaire, sont ainsi impliqués dans les actions sécuritaires à but préventif, parfois en inscrivant cette action dans le cadre du projet ESD (Education for Sustainable Development $)^{44}$. L'objectif de ce plan consiste alors à mettre l'accent sur le sens des responsabilités, ainsi que sur le respect dans la relation avec les autres et avec l'environnement local. Dans ce cadre, les enfants sont invités à éviter les endroits peu exposés et souvent dégradés; ils participent en même temps au nettoyage du quartier qui a souvent lieu durant le cours d'éducation morale (dōtoku). Ces actions ont pour objectif de faire prendre conscience aux enfants que leur bien-être dépend de tous, chacun contribuant à créer un environnement empêchant les infractions, y compris leurs voisins, notamment des personnes âgées qui travaillent pour le quartier. Notons que ce projet recouvre une dimension morale, fondées sur l'apprentissage de ce que les institutions scolaires appellent la tradition et la culture japonaises. Ce programme éducatif sera d'ailleurs renforcé en vue des Jeux olympiques, prévus pour 2020 et reportés d'un an. Comme le montre l'extrait suivant, nous observons là une forme de revalorisation du passé :

«Tout au départ, l'éducation a commencé à Terakoya ${ }^{45}$. C'est la constitution de la communauté qui en était le point de départ. Les enfants appartiennent à des familles du quartier. On redécouvre le quartier du point de vue des enfants. Pour les enfants, il y a des choses qu'ils apprennent non seulement avec les instituteurs, mais aussi avec les adultes du quartier. Et puis ils sont très contents, voire excités, quand ils dessinent des plans parce qu'ils se sentent utiles pour le quartier. " (Les plans sont distribués dans chaque foyer).

(Entretien avec une directrice d'école primaire, le 23 février 2016)

Dans les zones résidentielles étudiées, les actions préventives ne se résument ainsi pas seulement au repérage de dangers dans l'environnement urbain immédiat mais relèvent d'une dimension d'éducation morale, accordant une place importante aux aînés au sein du quartier. Ceci contraste avec les quartiers dans lesquels l'attention se porte davantage sur la fréquentation par des personnes n'y habitant pas, comme nous l'avions observé à Tokyo dans le quartier de Shibuya (voir section consacrée aux patrouilles d'habitants et aux voitures à gyrophare).

\section{Conclusion}

David Bayley et Clifford Shearing (2001) avaient remarqué une multilatéralisation du maintien de l'ordre montrant que les États n'en sont plus les seuls commanditaires et que des acteurs non-gouvernementaux contribuent aujourd'hui à la police, rappelant l'importance d'une distinction entre ceux qui conçoivent le maintien de l'ordre et ceux qui le mettent en œuvre. Au Japon, en mettant en avant la crainte de transformations sociales liées à l'urbanisation ainsi que celle des organisations criminelles menaçant la sécurité, l'État a envisagé un renforcement du maintien de l'ordre au niveau local, en l'inscrivant également dans une politique de prévention des catastrophes naturelles ainsi que d'animation du quartier et d'éducation morale des enfants et des jeunes. Cette politique s'inscrit dans le registre de la prévention situationnelle, et comprend deux volets qui sont liés: l'aménagement des espaces publics et différents dispositifs permettant la participation des habitants au maintien de l'ordre et à la tranquillité publique. 

sur les lieux non sécurisés dans leur environnement ; ils ont également eu pour effet de redéfinir le pouvoir local de certains habitants. Valorisés par leur participation au maintien de l'ordre et au repérage des risques dans leur quartier, ces habitants ont finalement endossé le rôle de responsables de communauté de quartier, qui exercent une action d'éducation morale. En cela, ils répondent aux attentes de l'Agence nationale de la police qui entend lutter contre l'affaiblissement des liens tant sur le plan familial qu'à l'échelle du voisinage. À la différence du modèle anglophone de prévention situationnelle dont s'est inspiré l'État japonais, les habitants participant au maintien de l'ordre ont accordé de l'importance à l'aspect éducatif, en s'alignant davantage sur une doctrine répétée depuis les années 1970 et focalisée sur le rôle clef du voisinage dans le contrôle social et la prévention des désordres. Le cas japonais contraste également avec le cas français, où la participation des habitants est encore envisagée avec beaucoup de méfiance par les acteurs institutionnels de la sécurité malgré des évolutions récentes.

La vigilance des habitants participant aux actions préventives se dirige généralement vers les personnes ne respectant pas les codes de comportement admis dans le quartier, qui ne correspondent pas aux normes instituées. Les individus sont finalement jugés « à risque » en fonction de leur degré d'intégration au quartier : l'espace du quartier tend alors à se refermer sur lui-même. Le pouvoir conféré aux habitants participe ainsi d'une forme de "discipline ", au sens foucaldien du terme, faisant " "marcher" un pouvoir relationnel qui se soutient lui-même par ses propres mécanismes» (Foucault, 1975). Autrement dit, sans sous-estimer les pratiques des habitants dont l'objectif de tri social varie en fonction des problématiques propres à chaque quartier, on peut dire que la visée d'un tel dispositif technique consiste, essentiellement, à responsabiliser habitants et organisations locales, sans que l'État ne montre explicitement, dans les espaces du quotidien, le contrôle qu'il exerce.

\section{BIBLIOGRAPHIE}

AGAMBEN G. (2014), Qu'est-ce qu'un dispositif?, Paris, Rivages poches, 80 p.

AMEMIYA M. et. al. (2006), Nihon ni okeru bōhan machi-zukuri e no hihanron no kōzō : 1998 nen ikō ni arawareta gensetsu wo taishō ni日本における防犯まちづくりへの批判論の構造 - 1998年 以降に現れた言説を対象に (Une analyse des critiques formulées après 1998 à l'encontre de la "fabrique de la ville pour la prévention de la délinquance" au Japon), Reports of the City Planning Institute of Japan, no 4, février, 2006.

BAUVET S. (2014), « Politiques sécuritaires et liens sociaux. Les implications d'un modèle contemporain de "participation citoyenne" ", in Serge Paugam (éd.), L'intégration inégale : Force, fragilité et rupture des liens sociaux, Paris, PUF, pp. 379-396.

DOI : 10.3917/puf.paug.2014.01.0379

Carnets de géographes, 15 | 2021 
BAYLEY D., SHEARING C. (2001), The New Structure of Policing: Description, Conceptualization, and Research Agenda, Washington (D. C.), National Institute of Justice.

CLIFFORD W. (1976), Crime Control in Japan, Lexington, MA, Lexigton Books.

DONZELOT J., MEVEL C., WYVEKENS A., (2003), Faire société. La politique de la ville aux États-Unis et en France, Paris, Le Seuil.

HASSENTEUFEL P. et MAILLARD (de) J. (2013), « Convergence, transferts et traduction. Les apports de la comparaison transnationale ", Gouvernement et action publique, vol. 3, no. 3, pp. 377-393.

FURSTENBERG F. (1971), Public Reaction to Crime in the Streets. The American Scholar. DOI : 40. 601-610

FOUCAULT M. (1975), Surveiller et punir, Paris, Gallimard.

FOUCAULT M. (1977), « Le jeu de Michel Foucault », entretien avec D. Colas, A. Grosrichard, G. Le Gaufey, J. Livi, G. Miller, J. Miller, J.-A. Miller, C, Millot, G. Wajeman, Ornicar, Bulletin Périodique du champ freudien, no. 10, juillet 1977, pp. 62-93. (Repris in Dits et Ecrits, II, 1976-1979, Paris, Gallimard, 1994, texte ${ }^{\circ} 206$, p. 299).

GERMES M. (2014), « Cartographies policières : la dimension vernaculaire du contrôle territorial », EchoGéo, no. 28, avril 2013/juin 2014.

DOI : $10.4000 /$ echogeo.13856

FUNG A. (2000) « Accountable Autonomy: Toward Empowered Deliberation in Chicago Schools and Policing », KSG Working Paper, 00-008, septembre 2000.

JOBARD F., MAILLARD J. (de) (2015), Sociologie de la police, Politiques, organisations, réformes, Paris, Armand Colin.

JOHNSTON L. (1996), « What is Vigilantism? », British Journal of Criminology, no. 36, vol. 2, pp. 423-452.

MAILLARD J. (de) (2009), « Réformes des polices dans les pays occidentaux. Une perspective comparée ", Revue française de science politique, vol. 59, no. 6, pp. 1197-1230.

MAILLARD J. (de) (2013), « Le difficile renouvellement des métiers de la sécurité publique. Le cas des correspondants de nuit parisiens », Criminologie, vol. 46, no. 2.

DOI : 10.7202/1020989ar

MALOCHET V. (2017), « Contours et positionnement d'une forme hybride de policing résidentiel », Champ pénal/Penal field, vol. XIV, [en ligne].

DOI : 10.4000/champpenal.9622

MARY P. (2001), « Pénalité et gestion des risques : vers une justice "actuarielle" en Europe ? ", Déviance et Société, vol. 25, no. 1, pp. 33-51.

MOSSER S. (2007), « Éclairage et sécurité en ville : l'état des savoirs », Déviance et Société, vol. 31, no. 1 , pp. $77-100$.

DOI : $10.3917 /$ ds.311.0077.

NAKATA M. (2000), Sekai no jūmin soshiki 世界の住民組織 (Les communautés locales dans le monde), Tokyo, Jichitai kenkyūsha 自治体研究社, p. 287.

OBLET T. (2008), Défendre la ville. La police, l'urbanisme et les habitants, Paris, PUF.

READ B. L., PEKKANEN R. (2009), Local Organizations and Urban Governance in East and Southeast Asia: Straddling state and society, Abingdon-on-Thames, Routledge. 
ROCHÉ S. (2000), « La théorie de la “vitre cassée” en France. Incivilités et désordres en public », Revue française de science politique, 50e année, no. 3, pp. 387-412.

SHIMIZU Masahiko清水雅彦. (2007), Chian seisaku to shite no « anzen anshin machi-zukuri » - kanshi to kanri no shōhai 治安政策としての「安全・安心まちづくり」一監視と管理の招牌 (《La fabrique d'une ville sûre et paisible » dans le cadre de la politique de sécurité : le tableau de surveillance et de gestion), Tokyo, Shakai hyōronsha 社会評論社, 333 p.

SKOGAN W. (2006), Police and Community in Chicago, a tale of three cities, Oxford, Presses de l'Université d'Oxford.

SIBLEY D. (1995), Geographies of exclusion: society and difference in the West, London/New-York, Routledge.

THOMANN B. (2017), «Les relations du travail pendant les années de la Haute Croissance », in M. Lucken, A. Bayard-Saka, E. Lozerand (dir.), Sengo, le Japon après la guerre, Paris, Presses de l'Inalco. DOI : 10.4000/books.pressesinalco.2424

TSUJINAKA Yutaka辻中豊 et al. (2009), Gendai nihon no jichikai chōnaikai 現代日本の自治会・町内 会 (Les jichikai et les chōnaikai dans le Japon contemporain), Tokyo, Bokutakusha 木鐸社, 259 p.

YAMAMOTO T. (2005), Bōhan machi-zukuri. Kodomo sumai chiiki wo mamoru 防犯まちつ"くり子 と“も・住まい・地域を守る (La fabrique de la ville pour la prévention de la délinquance. Protéger les enfants, le domicile, le quartier), Tokyo, Gyōsei き”ょうせい, p. 205.

WATANABE O. (2005), « Gurōbaruka. “Tsuyoi kokka” seisaku to gendai keisatsu no nerai » (Globalisation, politiques de l'« État fort » et enjeux policiers d'aujourd'hui), in Ogura Toshimaru 小倉利丸 (dir.), グローバル化と監視警察国家への抵抗 (Globalisation et résistance contre l'État de surveillance policière), Tokyo, Kinohanasha 樹花舎, pp. 13-118.

\section{NOTES}

1. Livre blanc de l'Agence nationale de la police (Keisatsu hakusho 警察白書), 1979.

2. Livre blanc de l'Agence nationale de la police (Keisatsu hakusho 警察白書), 1996.

3.

4. Le terme de dispositif est à entendre ici au sens foucaldien, comme « un ensemble résolument hétérogène, comportant des discours, des institutions, des aménagements architecturaux, des décisions réglementaires, des lois, des mesures administratives, des énoncés scientifiques, des propositions philosophiques, morales, philanthropiques, bref : du dit, aussi bien que du non-dit " (Foucault, 1977). Nous observerons en effet, dans le présent article, comment la mise en place effective d'aménagements dans les espaces urbains s'accompagne d'un discours de l'administration policière japonaise. Voir également, dans la même perspective, l'ouvrage de Giorgio Agamben, Qu'est-ce qu'un dispositif? (Agamben, 2014)

5. Ces communautés de quartier se caractérisent par leur existence dans un périmètre donné, qui exclut l'existence d'autres communautés de quartier au sein de cette même zone. Les résidents de ce périmètre partagent donc intérêts et préoccupations, et sont amenés à résoudre des problèmes locaux en s'entraidant. Ainsi, l'adhésion de tous les résidents de ce périmètre est en principe exigée (Tsujinaka et al., 2009: 18). Comme l'ont montré Read et Pekkanen (2009), la fonction de ces organisations d'habitants au Japon, comme en Corée ou en Chine, est d'agir en complément des autorités publiques, tout en travaillant au bénéfice de la vie quotidienne des habitants. On nomme "straddle» cette forme de gestion reposant sur deux centres d'action, même si les chōnaikai ne sont pas totalement assujettis au pouvoir politique (Read, Pekkanen, 
2009). Dès lors, si l'adhésion n'est pas formellement obligatoire, les résidents sont vivement invités à y adhérer. Cependant, on observe une indéniable baisse du taux d'adhésion : jusqu'au milieu des années 1990, plus de $60 \%$ des majeurs y adhéraient, alors que le taux a baissé jusqu'autour de $40 \%$ en 2007 (ibid., 27). Cette baisse de taux s'observe dans les grandes villes, où se concentrent les grands ensembles ou des commerces. (ibid., 205).

6. Keisatsu hakusho 警察白書 (Livre blanc de l'Agence nationale de la police), 1976.

7. Keisatsu hakusho 警察白書 (Livre blanc de l'Agence nationale de la police), 1984.

8. Cette période a été particulièrement médiatisée en raison d'augmentations successives du taux d'arrestation des mineurs. Dans un contexte où le taux de scolarisation au lycée dépassait $90 \%$ et que la majorité de population (plus de $90 \%$ ) se considéraient comme appartenant à la classe moyenne, l'administration policière soutenait notamment qu'il s'agissait d'une délinquance par «amusement» (asobigata hikō) provoquée par un manque de normes chez les jeunes et un dysfonctionnement des institutions éducatives.

9. Chōju shakai taisaku taikō 長寿社会対策大綱 (Bilan des politiques publiques face à une société vieillissante), 6 juin 1986.

10. Une telle approche se distingue par exemple de modèles de prévention sociale cherchant à agir sur les causes profondes de la délinquance.

11. W. Skogan (2006) a retenu, concernant le community policing, trois principales caractéristiques : 1) La décentralisation, 2) L'implication des communautés, 3) La résolution des problèmes. On retrouve ces trois éléments dans le cas japonais.

12. Le livre blanc de l'Agence nationale de la police de 1994 cite par exemple le "Community police officer program ", développé dans la ville de New-York à partir de 1984, les actions de "CITIZENS (Commitee For New York)» et le "San Francisco SAFE (Safety Awareness For Everyone) », ou encore l'installation des «Sur Station » dans la ville de Philadelphie.

13. Il s'agit notamment des Neighborhood Foot Patrol Program.

14. Safer Cities Project, Special Constable.

15. Neighborhood Police Post.

16. À titre exemple, la tolérance zéro a été mise en avant par Jacques Chirac, lors de la campagne présidentielle de 2002, afin de désigner plutôt une forme d'impunité zéro c'est-à-dire une réponse avant tout judiciaire au lieu d'une simple répression policière (Maillard et Le Goff, 2009). Voir également les travaux de Jacques de Maillard sur le dispositif des correspondants de nuit (Maillard, 2013), sur la participation citoyenne (Bauvet, 2014) ou encore sur le Groupement parisien inter-bailleurs de surveillance (Malochet, 2017).

17. Le tremblement de terre de Hanshin-Awaji a touché le sud du département de Hyōgo à 5 h 46, le 17 janvier 1995. La magnitude était de 7,3 sur l'échelle de Richter et le tremblement de terre fit plus de 6400 morts et environ 43800 blessés. Près de 640000 logements furent détruits, parmi lesquels environ 7500 furent incendiés. Il faut noter que, lors de cette catastrophe, la majorité des personnes décédées l'ont été à cause de l'effondrement de bâtiments ou par suffocation. Afin de prévenir des infractions (cambriolages, escroquerie, développement d'actes commis par des organisations criminelles) après ce séisme, des habitants se sont ainsi organisés entre eux, notamment en réalisant des patrouilles (comptant environ 570 participants par jour), en plus des patrouilles réalisées par 3830 bénévoles venant des sociétés de sécurité privée et des associations de prévention de la délinquance. L'Agence nationale de la police a alors souligné l'importance de ces patrouilles qui auraient été mises en place en collaboration avec les policiers dans les zones touchées par le sinistre, afin d'assurer la sécurité des biens dans les logements détruits mais aussi afin d'apporter une forme 
d'écoute aux victimes exprimant des besoins, notamment à travers des distributions de nourriture, des soins, la communication d'informations, etc.

18. Le lundi 20 mars 1995, à Tokyo, du gaz sarin a été diffusé, aux heures de pointe, dans des wagons de métro passant par la station de Kasumigaseki, zone où se concentrent les administrations centrales. Le bilan fut de 13 morts et plus de 5800 blessés. Du gaz sarin avait été également diffusé dans un quartier résidentiel de la ville de Matsumoto (Nagano), le 27 juin 1994, ce qui fit 8 morts et plus de 140 blessés. Ces attentats furent tous deux commis par la secte Aum Shinrikyō.

19. Keisatsu hakusho 警察白書 (Livre blanc de l'Agence nationale de la police), 1996.

20. Fondée en 1962 sous tutelle de l'Agence nationale de la police japonaise, cette fondation est devenue une fondation à intérêt public en 2012. Travaillant en partenariat avec la police, elle vise à diffuser les connaissances relatives aux actions préventives des habitants, que cela concerne la prévention de la consommation des drogues, l'éducation des adolescents, ou encore la lutte contre les organisations criminelles.

21. $\mathrm{CP}<h$, kun étant un suffixe utilisé notamment pour désigner les jeunes garçons.

22. Il s'agit d'un groupe de réflexion formé au sein du service de la sécurité publique (hoanbu) de l'Agence nationale de la police visant à réfléchir à la promotion des mesures préventives de la délinquance ainsi qu'au rôle de la police au niveau local. Le groupe était composé de spécialistes du droit administratif, de droit pénal, d'architectes, de cadres policiers ainsi que de représentants du Congrès national de parents et d'enseignants (Shimizu, 2007).

23. Cette catégorie d'« étrangers » n'inclut pas les résidents dotés d'un visa permanent (eijū visa 永住ビザ), les militaires états-uniens travaillant au Japon, ainsi que ceux dont le titre de séjour n'est pas clairement établi.

24. Keisatsu hakusho 警察白書 (Livre blanc de l'Agence nationale de la police), 2003.

25. Cette théorie, nommée "broken windows » en anglais, a été publiée en 1982 par J. Q. Wilson et G. Kelling, et veut que "dans le cas où une vitre cassée n'est pas remplacée, toutes les autres vitres connaissent bientôt le même sort ». L'idée est que, dès que se multiplient des signes d'abandon, le vandalisme tend à se manifester, suivi de comportements de vols et d'agressions (Roché, 2000, p. 388). Cette théorie a été largement répandue et répétée au sein des réunions policières mais aussi dans les médias et dans les cours de formation des bénévoles participant aux actions préventives. Ce projet détaille également cinq problèmes à résoudre dans les cinq années à venir : 1) la prévention de la délinquance menaçant la tranquillité de la vie quotidienne, 2) la prévention de la délinquance juvénile, 3) l'élimination de la menace venant de l'internationalisation du crime et des étrangers en situation illégale, 4) la lutte contre les organisations criminelles (crimes lié aux drogues, au trafic d'armes, aux activités des usuriers, à la cybercriminalité, etc.), 5) le renforcement des équipements et des systèmes : effectifs de la police, des procureurs, renforcement des contrôles à la frontière, amélioration du système de réinsertion après la sortie de prison, etc.

26. Ce ministère est aujourd'hui devenu le ministère du Territoire, des Infrastructures, des Transports et du Tourisme (kokudo kōtsūshō 国土交通省, MLIT).

27. Ces « villes modèles » ont été choisies suite à l'examen des actions recensées, ceci dans le but de diffuser ces modèles au niveau national. Les villes choisies peuvent bénéficier du soutien public à la fois sur un plan financier et en matière d'équipement matériel.

28. Ces quartiers ont été choisis à la suite du tremblement de terre de Hanshin-Awaji et de meurtres successifs dans la ville de Kobe.

29. Un grand nombre d'exemples anglais et nord-américains ont été présentés à l'Agence nationale de la police japonaise : le cas britannique du NHBC (National House- 
Building Council), établissant des critères de sécurité dans les immeubles (qualité des portes, des fenêtres, des clés mais aussi du design de la ville, etc.); le projet, britannique également, "Secured by Design »; l'activité spécifique d'agents tels que les "crime prévention design advisor» ou encore les "architectural liaison officer». Ils ont également envisagé d'importer l'idée d'un service du SCAS, unité de la police britannique spécialisée dans l'analyse des infractions et établissant des bases de données visant à détecter l'émergence potentielle de foyers de délinquance. Le rapport cite également la circulaire interministérielle de 1984 et le «Crime \& Disorder Act » de 1998, qui faisaient appel aux efforts de la communauté en collaboration avec les collectivités locales et la police, ou encore les "Crime Prevention Design Advisors", dont la formation a commencé en 1986 à Londres, qui ont pour mission de dispenser des conseils relatifs à la prévention de la délinquance, en vérifiant les plans de développement urbain en fonction des réalités locales.

30. Extrait d'un résumé de la deuxième réunion de réflexion sur les techniques pour la «Fabrique de la ville (machi-zukuri) sûre (anzen) et paisible (anshin) ».

31. Les conclusions des réunions ont été publiées dans le Livret de la fabrique de la ville sûre et paisible - Community \& Urban Design for Crime Prevention (présentant une traduction en anglais), Gyōsei, mars 2001, réunion de réflexion sur « la fabrique d'une ville sûre et paisible ».

32. Il s'agit de la zone environnant la gare de la ville d'Iwami (Hokkaidō), des centres-villes de Furukawa (Miyagi), de Tsuruoka (Yamagata), de Jōetsu (Nīgata), de Toyama (Toyama), d'Okinawa (Okinawa), du quartier résidentiel de la ville de Kasugai (Aichi), de Toyonaka (Ōsaka), Zentsūji (Kagawa) ainsi que d'un quartier populaire de l'arrondissement de Sumida (Tokyo).

33. Les effets de l'éclairage ne sont en revanche pas constatés scientifiquement, comme le montrent les travaux de Mosser (2007).

34. «Quartiers faisant l'objet du Projet de modèle pour fabriquer une ville où l'on peut vivre à pied ", 2001. Ce projet est le fruit d'une réunion interministérielle impliquant notamment le bureau du Premier ministre, les ministères de la Construction, de l'Environnement, des Finances, de l'Éducation, de la Santé, de l'Agriculture, des Transports, des Collectivités territoriales, ainsi que l'Agence nationale de la police.

35. Il s'agit d'enquêtes sur l'aménagement urbain des quartiers résidentiels autour des écoles qui ont été réalisées en 2002 à l'échelle interministérielle.

36. Le recensement repose sur la prise en compte des groupes de plus de cinq personnes réalisant au moins une activité notable par mois à l'exception des réunions.

37. Livre blanc de l'Agence nationale de la police, 2004.

38. Journal d'activités d'un groupe d'habitants, juillet 2008.

39. Ce droit demeure réservé aux structures publiques ou privées qui procèdent à des patrouilles dans une visée exclusivement préventive. Les utilisateurs de ce gyrophare doivent au préalable déposer une demande au commissariat et, après obtention d'un certificat, l'envoyer au responsable régional des transports. Dans la plupart des cas, les demandes émanent d'associations en partie financées par la police.

40. $161^{\text {e }}$ débat de la Chambre des conseillers (sanguiin), 2 novembre 2004, propos du chef du bureau de la sécurité du quotidien à l'Agence nationale de la police.

41. "Sur le renforcement des mesures à l'égard de ceux que l'on qualifie de sans-abri ", communiqué de l'Agence nationale de la police, 26 mai 1999.

42. Cette application, « Digi police», mise en place en 2016 par la police métropolitaine de Tokyo, permet également aux individus de déclencher deux types d'alertes : l'une émet un son aigu et 
l'autre énonce les phrases "Je suis agressé(e). Au secours" (Chikan desu. Tasukete kudasai). L'application permet également d'envoyer un mail automatique à une adresse pré-enregistrée par exemple, celle des parents d'un enfant. Cela leur permet de connaître le lieu où l'alerte a été déclenchée. Cette application a notamment pour fonction de diffuser des informations ou d'être utilisée comme alarme, mais elle ne se sert pas à recueillir des informations.

43. Ces détails proviennent d'un rapport intitulé « Examen des moyens pour veiller sur les enfants par les techniques ubiquitaires », bureau de communication générale de Kyūshū du ministère des Affaires générales (Sōmushō), juillet 2007.

44. Ce projet, élaboré à partir du rapport de l'ONU «Our Common Future » de 1986, a été proposé à l'occasion du sommet de Johannesburg sur le développement durable de 2002. Mis en œuvre entre 2005 et 2014 sous l'égide de l'Unesco, il avait pour objectif de diffuser les principes, les valeurs et des pratiques en faveur du développement durable. Au Japon, ce projet a été réalisé en mars 2006 en l'adaptant au contexte national. Il visait alors à promouvoir les réflexions sur l'écologie et le développement durable. Voir : « Proposition relative à l'utilisation d'Unesco school pour la promotion et la diffusion de l'ESD » (Education for Sustainable Development), février 2008, sous-comité pour l'éducation dans la commission nationale de l'Unesco au Japon.

45. Il s'agit d'une institution scolaire destinée aux enfants de la classe populaire, ayant pour objectif de leur apprendre l'écriture ou des calculs élémentaires. Leur enseignement se diffusa au milieu du XVIII ${ }^{\mathrm{e}}$ siècle et était notamment assuré par les moines, les guerriers (bushi), les prêtes shintoïstes ou les médecins.

\section{RÉSUMÉS}

Craignant une baisse de confiance à l'égard des policiers ainsi qu'un affaiblissement des liens sociaux au sein des communautés locales en raison d'une urbanisation croissante du pays, la police japonaise s'est attachée à renforcer localement ses partenariats avec la population, notamment à partir des années 2000. Dans cette perspective, l'État japonais a orienté l'aménagement des espaces publics en s'inspirant de la prévention situationnelle, et a encouragé le développement de différentes technologies de surveillance. Ceci a conduit à la mise en place d'un maillage dense de réseaux de surveillance dans les espaces publics, sous couvert de «lien social» et de "protection» des citoyens, non seulement dans des quartiers commerciaux marqués par une forte fréquentation de clients extérieurs, mais également dans des quartiers résidentiels où les commerces locaux sont souvent fréquentés par des habitants du quartier. Des enquêtes de terrain ainsi que l'étude de documents législatifs produits depuis les années 1970 montrent à cet égard que les actions préventives réalisées par les habitants comportent un aspect d'éducation morale à destination des enfants. Les technologies de surveillance ont ainsi contribué à la légitimation d'une surveillance par les habitants, exerçant une forme de tri social au sein des quartiers.

Concerned about a decline in confidence in the police and a weakening of social ties as a result of increasing urbanization, the Japanese police has focused on strengthening their local partnership with the general public, particularly from the 2000s onwards. Inspired by situational crime prevention, the Japanese State has oriented the planning of public spaces in this perspective and 
encouraged the development of surveillance technologies. This led to the development of surveillance networks in public spaces under the cover of "social bonding" and "protection", not only in commercial districts marked by a high number of visitors, but also in residential areas where local shops are often frequented by local residents. Fieldwork research and the study of legislative documents produced since the 1970s show that the preventive actions conducted by inhabitants also include an aspect of moral education directed at children, particularly in periurban areas. Surveillance technologies thus led to the legitimization of surveillance by the inhabitants, exercising a form of screening within the neighborhood.

\section{INDEX}

Thèmes : Carnets de recherches

Keywords : Neighborhood, Prevention, Surveillance, Public Space, Police, Participation, Inhabitants, Citizenship

Mots-clés : prévention situationnelle, surveillance, espace public, police, participation, habitants, citoyenneté

\section{AUTEUR}

\section{NAOKO TOKUMITSU}

Maître de conférences en études japonaises (sociologie), Inalco.

naoko.partiot[at]inalco.fr 